\title{
Fatores influenciadores da esperança nos pais de crianças com doença crónica
}

Factors influencing hope in parents of children with chronic illness

Factores que influyen en la esperanza de los padres de niños con enfermedad crónica

\author{
Telma Luisa Ferreira Duarte Maravilha' ${ }^{1}$ nttps://orcid.org/0000-0001-7637-4306 \\ Mónica Filipa Louro Marcelino² Đ nttps://orcid.org/0000-0002-1273-041X \\ Zaida Borges Charepe ${ }^{3}$ io hitps://orcid.org/0000-0003-0080-4482
}

Como citar: Maravilha TL, Marcelino MF, Charepe ZB. Fatores influenciadores da esperança nos pais de crianças com doença crónica. Acta Paul Enferm. 2021;34:eAPE001545.

DOI

http://dx.doi.org/10.37689/actaape/2021AR01545

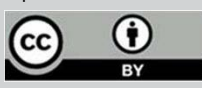

Descritores

Confiança; Criança; Doença crónica; Espiritualidade; Apôio social; Pais

Keywords

Hope; Child; Chronic disease; Spirituality; Socia support; Parents

Descriptores

Confianza; Niño: Enfermedad crónica; Espiritualidad; Apoyo social; Padres

Submetido 20 de Junho de 2020

Aceito

3 de Março de 2021

Autor correspondente

Telma Luisa Ferreira Duarte Maravilha

E-mail: telma_sb_17@hotmail.com

\section{Resumo}

Objetivo: Mapear na literatura científica estudos que conduzam à identificação dos fatores que influenciam a vivência de esperança em pais de crianças com doença crónica.

Métodos: Realizada uma revisão da literatura do tipo Scoping Review, seguindo a metodologia proposta pelo Joanna Briggs Institute, nas bases de dados Pubmed, CINHAL Plus with Full texto e na MEDLINE Plus with Full text, no intervalo cronológico compreendido entre 2009 e 2019, para responder à questão: quais os fatores que influenciam a vivência de esperança em pais de crianças com doença crónica?

Resultados: Após aplicação dos critérios de inclusão e exclusão, foram selecionados dez estudos, dos quais se depreendeu que a esperança é um recurso interno positivo e motivador e que está orientada para 0 presente e para o futuro. Existem fatores promotores de esperança, como o foco nos pontos positivos, fé/ religião e apoio social; e fatores ameaçadores à esperança parental, como o esgotamento físico e emocional, negativismo percecionado por parte dos outros, medo e incerteza.

Conclusão: A esperança parental é influenciada pelo estado de saúde dos filhos, eficácia dos tratamentos e contexto psicossocial, tendo um papel significativo na adaptação dos pais à condição de saúde da criança.

\section{Abstract}

Objective: To map in scientific literature studies that lead to the identification of factors that influence the experience of hope in parents of children with chronic illness.

Methods: A scoping review literature review was carried out, following the methodology proposed by the Joanna Briggs Institute in the PubMed, CINHAL Plus with Full text and MEDLINE Plus with Full text databases, in the chronological interval between 2009 and 2019, to respond to the question: what are the factors that influence the experience of hope in parents of children with chronic illness?

Results: After applying the inclusion and exclusion criteria, ten studies were selected, from which it appeared that hope is a positive and motivating internal resource and that it is oriented towards the present and the future. There are factors that promote hope, such as the focus on positives, faith/religion and social support; and factors that threaten parental hope, such as physical and emotional exhaustion, perceived negativity on the part of others, fear and uncertainty.

Conclusion: Parental hope is influenced by children's health status, effectiveness of treatments and psychosocial context, playing a significant role in adapting parents to children's health condition. 


\section{Resumen}

Objetivo: Mapear en la literatura científica estudios que conduzcan a la identificación de los factores que influyen en la vivencia de esperanza de los padres de niños con enfermedad crónica.

Métodos: Se realizó una revisión de la literatura tipo Scoping Review, siguiendo la metodología propuesta por el Joanna Briggs Institute, en la base de datos Pubmed, CINHAL Plus with Full texto y en la MEDLINE Plus with Full text, en el período cronológico comprendido entre 2009 y 2019 para responder la pregunta: ¿qué factores influyen en la vivencia de esperanza de los padres de niños con enfermedad crónica?

Resultados: Luego de aplicar los criterios de inclusión y exclusión, se seleccionaron diez estudios, de los cuales se dedujo que la esperanza es un recurso interno positivo y motivador y que está orientada hacia el presente y el futuro. Existen factores que promueven la esperanza, como el énfasis en los puntos positivos, la fe/religión y el apoyo social; y factores que amenazan la esperanza parental, como el agotamiento físico y emocional, el negativismo percibido por parte de los otros, el miedo y la incertidumbre.

Conclusión: La esperanza parental está influenciada por el estado de salud de los hijos, la eficacia de los tratamientos y el contexto psicosocial, factores que tienen un papel significativo en la adaptación de los padres a la condición de salud del niño.

\section{Introdução}

A criança é o foco dos cuidados pediátricos, no entanto os pais também são assumidos como parte integrante do cuidar. ${ }^{(1)}$ A doença crónica pode ser definida como uma doença de longa duração, com incapacidades e défices residuais a médio e longo prazo, expresso em maior ou menor percentagem de necessidade de cuidados de saúde e sua supervisão. ${ }^{(2)}$

A esperança é um conceito abordado há várias décadas e em diferentes áreas científicas, no entanto, o reconhecimento desta como fenómeno essencial à disciplina de enfermagem e, sobre o qual os estudos necessitam de recair é mais recente. ${ }^{(3)}$ Enquanto conceito tem emergido com crescente interesse e gradual reconhecimento, sendo definida como uma experiência de sentido e propósito para a vida, ${ }^{(4)}$ com carácter multidimensional e de grande complexidade. ${ }^{(5)}$ Algumas teorias desenvolvidas no âmbito da disciplina de Enfermagem, referem que existem quatro elementos que definem a esperança de forma processual, a saber: processo experimental, espiritual, racional e relacional. ${ }^{(5)}$ Esta é um fenómeno caraterizado pela possibilidade da pessoa se responsabilizar pela sua própria existência, dirigir a sua vida e transcender os aspetos simples da realidade. ${ }^{(6)}$

Devido ao seu caráter individual e multidimensional, a esperança é essencial no processo de saúde-doença. Esta contribui para a capacitação da pessoa para enfrentar momentos de crise, para manter a qualidade de vida, contribuindo para a promoção de saúde e determinação de objetivos realistas, assumindo-se como um dos aspetos centrais no cuidado de enfermagem. ${ }^{(7)}$ A esperança pode ainda ser expe- rienciada como conforto, e como uma possibilidade de sair de um ciclo de sofrimento. ${ }^{(7)}$

Apesar da investigaçáo desenvolvida e do interesse crescente pelo tema, ainda são escassos os estudos que abordam a vivência de esperança em pais de crianças com doença crónica. ${ }^{(6)}$ A exemplo, o estudo de Charepe ${ }^{(8)}$ analisou a influência dos "(...) grupos de ajuda mútua no desenvolvimento da esperança em pais de crianças com doença crónica” ${ }^{(8-13)}$ Outros autores conferiram-lhe enfoque como um recurso essencial e sustentador da vida na perspetiva dos pais, em contextos de cuidados às crianças com doença crónica, incurável e potencialmente fatal. ${ }^{(6,9,10)}$

Sisk, Kang \& $\operatorname{Mack}^{(10)}$ num estudo realizado com pais de crianças com o diagnóstico clínico de doença oncológica, concluem que as fontes de esperança parental ultrapassam a esperança na cura, sendo determinante perceber como a mesma persiste durante a trajetória de doença da criança com mau prognóstico.

Os cuidados que visam a promoção da esperança assumem deste modo um papel relevante atendendo à natureza da doença crónica, às limitaçóes e aos desafios impostos pela gestáo quotidiana da sintomatologia, do regime terapêutico e às necessidades de cuidados de saúde e recursos disponíveis.

Atendendo à dispersão da literatura científica acerca da esperança parental, torna-se importante clarificar as definiçóes e limites concetuais através da pesquisa existente. Desta forma, evidencia-se a relevância e a pertinência da realização de uma scoping review, com o objetivo de mapear na literatura científica estudos que conduzam à identificação dos fatores que influenciam a vivência de esperança, em pais de crianças com doença crónica. 
Foi efetuada uma pesquisa preliminar das revisóes sistemáticas publicadas sobre esta temática, nas seguintes bases de dados, a saber: PROSPERO, Cochrane Database of Systematic Reviews, JBI Evidence Synthesis, PubMed e CINAHL e não foram identificadas quaisquer revisóes sistemáticas atuais ou em curso sobre o tema. Esta pesquisa foi realizada a $12 \mathrm{de}$ janeiro de 2020 e repetida a 20 de fevereiro de 2020 .

\section{Métodos}

A presente revisão da literatura utilizou o método descrito por The Joanna Briggs Institute (JBI). ${ }^{(1)}$

A formulação da questão de revisão, quais os fatores que influenciam a esperança em pais de crianças com doença crónica? teve por base a mnemónica PCC (participantes - pais de crianças com doença crónica; conceito - vivências de esperança; contexto - qualquer contexto de cuidados de saúde pediátricos, no hospital, nos cuidados primários ou em casa). Cada acrónimo tornou-se decisivo para a orientação da revisão e construção da estratégia de pesquisa, de forma a obter informação científica fiável.

Quanto ao tipo de fontes foram incluídos estudos primários de natureza qualitativa, quantitativa ou mistos, publicados no intervalo cronológico 2009 e 2019, nos idiomas inglês, português e espanhol. Este friso temporal justifica-se dado que esta revisão parte de um trabalho de investigação de maior amplitude realizado no ano de 2009, que contemplou uma revisão sistemática acerca dos fatores promotores de esperança parental. ${ }^{(8)}$

Os critérios de exclusão respeitados foram: estudos sobre pais de crianças com doença aguda, pais com filhos adultos, pais de crianças prematuras e/ou com alteraçóes do comportamento, dadas as especificidades das suas condiçôes clínicas.

$\mathrm{Na}$ pesquisa utilizou-se a estratégia em três etapas. ${ }^{(12)}$ Inicialmente foi realizada pesquisa nas bases de dados (MEDLINE with full text e CINAHL with full text), seguida de análise realizada por dois revisores independentes, das palavras contidas no título e resumo dos artigos selecionados assim como dos termos chave. Após a identificação dos descritores a utilizar constituiu-se e aplicou-se a equaçáo de pesquisa nas seguintes bases de dados, da plataforma eletrónica EBSCOhost: Cumulative Index to Nursing and Allied Health Literature Complete (CINAHL), MEDLINE Complete, Nursing \& Allied Health Collection: Comprehensive; Cochrane Central Register of Controlled Trials, Cochrane Database of Systematic Reviews, Cochrane Methodology Register; Library, Information Science \& Technology Abstracts (LISTA) e MedicLatina. Adicionalmente pesquisou-se nas plataformas PubMed, SciELO e Web of Science. Consultou-se de forma sistemática, a lista de referência bibliográficas dos artigos selecionados como forma de identificar artigos relevantes para esta revisão.

Após a pesquisa, todos os registos identificados foram agrupados para análise e removidos os artigos duplicados, com recurso ao software de gestão bibliográfica EndNote. Os dois revisores analisaram o texto completo dos artigos que cumpriam os critérios de inclusão. Em situação de discordância entre os revisores, foi efetuada a discussão baseada nos objetivos da revisáo com recurso a um terceiro revisor independente.

Foram incluídos 24 artigos, sendo que após análise criteriosa e aplicação dos critérios de inclusão e exclusão foram rejeitados 11. Restaram 13 estudos para avaliação crítica, os quais foram analisados e extraída a informação relevante para dar resposta à questão formulada. Depois da leitura e análise integral individualizada foram mantidos dez artigos científicos (Figura 1).

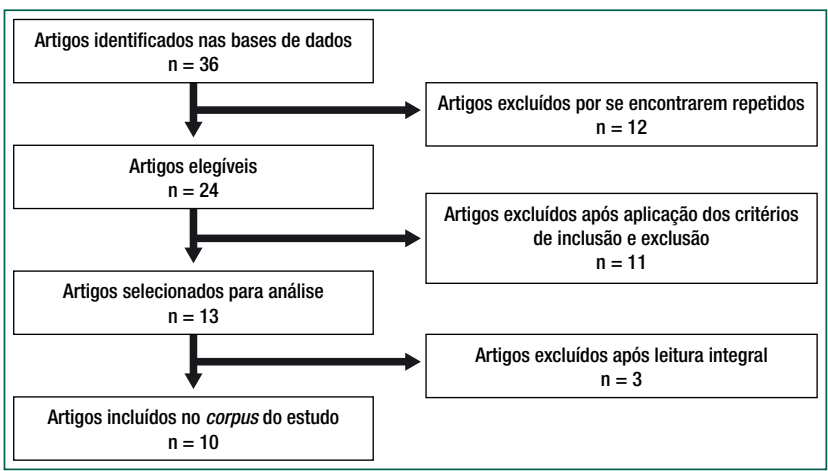

Figura 1. Fluxograma PRISMA ${ }^{(13)}$ do processo de seleção de estudos 


\section{Resultados}

Os resultados foram analisados de acordo com a questão de revisão e a natureza metodológica dos estudos. Foi realizada uma caraterização dos artigos incluídos na amostra teórica desta revisão (Quadro 1). ${ }^{(6,9,14-21)}$

Da análise dos dados concluiu-se: de 2010 a 2012 inclusive, e em 2017 e 2019 não se encontraram publicaçóes relevantes; 2013 foi o ano com maior número de publicaçóes pertinentes acerca do tema (três artigos publicados); em 2009 e 2015 foram publicados dois artigos por ano enquanto que em 2014, 2017 e 2018 registou-se um artigo.
Os estudos selecionados incidiram na relação da esperança com as vivências dos pais de crianças com doença crónica, verificando-se que seis estudos $(60 \%)$ seguiram uma metodologia qualitativa (paradigma indutivo), dois estudos (20\%) uma metodologia quantitativa (paradigma dedutivo), um estudo com recurso à abordagem metodológica de mixed methods (10\%) e uma revisão da literatura (10\%).

Os estudos que compuseram o corpus do estudo apresentaram dados significativos, que sustentam a conceptualização da esperança nas vivências dos pais de crianças com doença crónica, emergindo os fatores que a influenciam. Destes, destacam-se os fato-

Quadro 1. Caraterização dos estudos analisados na revisão de literatura

\begin{tabular}{|c|c|c|c|c|c|}
\hline $\begin{array}{l}\text { Autores / Ano } \\
\text { / País }\end{array}$ & Título & Objetivos & Tipo de Estudo & Resultados & Principais fatores \\
\hline $\begin{array}{l}\text { Samson et al }{ }^{(6)} \\
2009 \\
\text { Canadá }\end{array}$ & $\begin{array}{l}\text { The lived experience of } \\
\text { hope among parents of } \\
\text { a child with Duchenne } \\
\text { muscular dystrophy: } \\
\text { perceiving the human } \\
\text { being beyond the illness }\end{array}$ & $\begin{array}{l}\text { Descrever as vivências de esperança } \\
\text { entre os pais de crianças com Distrofia } \\
\text { Muscular de Duchenne (DMD), em } \\
\text { contexto específico de adaptação } \\
\text { psicossocial; } \\
\text { Descrever e compreender como é } \\
\text { que a esperança emerge na trajetória, } \\
\text { considerando o desfecho fatal. }\end{array}$ & \begin{tabular}{|l|} 
Qualitativo: \\
Abordagem \\
Fenomenológica
\end{tabular} & $\begin{array}{l}\text { A experiência parental de esperança emerge da } \\
\text { avaliação cognitiva da criança com DMD. } \\
\text { As perceções dos pais face à doença da } \\
\text { criança (grave perda; necessidade de } \\
\text { adaptação; experiência espiritual e intangível } \\
\text { que Ihes permitiu o reencontro com o filho), } \\
\text { conduzem a diferentes formas de esperança. }\end{array}$ & $\begin{array}{l}\text { Perceções dos pais acerca da } \\
\text { doença da criança. }\end{array}$ \\
\hline \begin{tabular}{|l|} 
Lloyd \& Hastings $^{(14)}$ \\
2009 \\
United Kingdom
\end{tabular} & $\begin{array}{l}\text { Hope as a psychological } \\
\text { resilience factor in } \\
\text { mothers and fathers of } \\
\text { children with intellectual } \\
\text { disabilities }\end{array}$ & $\begin{array}{l}\text { Explorar a relação da esperança como } \\
\text { um potencial de resiliência, no bem- } \\
\text { estar dos pais de crianças em idade } \\
\text { escolar com deficiência intelectual, } \\
\text { baseado na Teoria de Esperança de } \\
\text { Snyder. }\end{array}$ & Quantitativo & $\begin{array}{l}\text { A esperança foi medida compreendendo dois } \\
\text { componentes: a perceção de que se pode } \\
\text { alcançar os seus objetivos (metas) e que se } \\
\text { pode encontrar rotas alternativas para atingir os } \\
\text { mesmos, caso seja necessário (vias). }\end{array}$ & $\begin{array}{l}\text { Ansiedade e depressão no pai. } \\
\text { Afeto positivo no pai. } \\
\text { Bem-estar das mães. }\end{array}$ \\
\hline $\begin{array}{l}\text { Granek et al(15) } \\
2013 \\
\text { Israel }\end{array}$ & $\begin{array}{l}\text { Trajectory of parental } \\
\text { hope when a child has } \\
\text { difficult-to-treat cancer: } \\
\text { a prospective qualitative } \\
\text { study }\end{array}$ & $\begin{array}{l}\text { Perceber a esperança parental quando } \\
\text { uma criança com cancro se encontra } \\
\text { em tratamentos, depois do primeiro ano } \\
\text { após o diagnóstico. }\end{array}$ & \begin{tabular}{|l|} 
Qualitativo: \\
Grounded Theory
\end{tabular} & $\begin{array}{l}\text { A esperança parental muda ao longo do } \\
\text { primeiro ano após o diagnóstico. É considerada } \\
\text { um recurso interno positivo, motivacional que } \\
\text { orienta para o presente e futuro. } \\
\text { Foram identificadas duas categorias: esperança } \\
\text { orientada para o presente (não terem } \\
\text { sofrimento nem complicações) e esperança } \\
\text { orientada para o futuro (para a cura, para o } \\
\text { futuro, por um milagre e por mais tempo com a } \\
\text { criança em fim de vida). }\end{array}$ & $\begin{array}{l}\text { Progressão da doença. } \\
\text { Dor e sofrimento da criança. }\end{array}$ \\
\hline $\begin{array}{l}\text { Barrera et al(16) } \\
2013 \\
\text { Canadá }\end{array}$ & $\begin{array}{l}\text { The Tenacity and } \\
\text { Tenuousness of Hope }\end{array}$ & $\begin{array}{l}\text { Explorar a esperança parental durante } 0 \\
\text { processo de tratamento do cancro dos } \\
\text { seus filhos; } \\
\text { Identificar facilitadores e barreiras na } \\
\text { sua manutenção. }\end{array}$ & \begin{tabular}{|l|} 
Qualitativo: \\
Grounded Theory
\end{tabular} & $\begin{array}{l}\text { A esperança parental está relacionada com a } \\
\text { cura das crianças. O seu futuro encontra-se } \\
\text { geralmente dependente da resposta da criança } \\
\text { ao tratamento e ao contexto psicossocial. } \\
\text { Existem facilitadores e barreiras à manutenção } \\
\text { da esperança identificados pelos pais. }\end{array}$ & $\begin{array}{l}\text { Consciencialização para os aspetos } \\
\text { positivos ou negativos relacionados } \\
\text { com a doença da criança. } \\
\text { Pensamento positivo. } \\
\text { Efeitos do tratamento na criança. } \\
\text { Foco num presente positivo. } \\
\text { Espiritualidade. } \\
\text { Suporte psicossocial. } \\
\text { Sobrecarga de informação. } \\
\text { Negatividade de outros. } \\
\text { Esgotamento físico e emocional. } \\
\text { Medo e incerteza. }\end{array}$ \\
\hline \begin{tabular}{|l|} 
Markward, Benner \\
\& Freese ${ }^{(17)}$ \\
2013 \\
Colombia
\end{tabular} & $\begin{array}{l}\text { Perspective of parents on } \\
\text { Making Decisions about } \\
\text { the Care and Treatment } \\
\text { of a Child with Cancer }\end{array}$ & $\begin{array}{l}\text { Perceber a perspetiva dos pais em } \\
\text { relação à tomada de decisão acerca } \\
\text { do cuidado e tratamento da criança } \\
\text { com cancro. }\end{array}$ & \begin{tabular}{|l|} 
Revisão da \\
Literatura
\end{tabular} & $\begin{array}{l}\text { A esperança influência a tomada de decisão } \\
\text { dos pais em relação ao tratamento e cuidados } \\
\text { à criança com cancro. Ajuda na preparação } \\
\text { para os aspetos negativos do tratamento } \\
\text { e pode ser promovida através de uma } \\
\text { comunicação apropriada pelos profissionais } \\
\text { de saúde. É importante na tomada de decisão } \\
\text { em fim de vida. Para os pais a esperança é } \\
\text { um aumento do tempo e da qualidade de vida } \\
\text { dos filhos. }\end{array}$ & $\begin{array}{l}\text { Perceção de apoio. } \\
\text { Comunicação. } \\
\text { Informação e conhecimento. } \\
\text { Participação dos pais nos cuidados } \\
\text { à criança. } \\
\text { Qualidade de vida e bem-estar da } \\
\text { criança. } \\
\text { Sentido de controle. } \\
\text { Confiança. } \\
\text { Situação financeira. } \\
\text { Satisfação com os cuidados } \\
\text { recebidos. }\end{array}$ \\
\hline
\end{tabular}




\begin{tabular}{|c|c|c|c|c|c|}
\hline $\begin{array}{l}\text { Autores / Ano } \\
\text { / País }\end{array}$ & Título & Objetivos & Tipo de Estudo & Resultados & Principais fatores \\
\hline $\begin{array}{l}\text { Bally et al }{ }^{(9)} \\
2014 \\
\text { Canadá }\end{array}$ & Keeping Hope Possible & $\begin{array}{l}\text { Compreender a vivência de esperança } \\
\text { em pais que cuidam de crianças em } \\
\text { processo de tratamento contra o } \\
\text { cancro. }\end{array}$ & $\begin{array}{l}\text { Qualitativo: } \\
\text { Grounded Theory }\end{array}$ & $\begin{array}{l}\text { Esperança parental é definida como essencial, } \\
\text { deliberada, que sustenta a vida, dinâmica e } \\
\text { cíclica. O processo de esperança passa por } \\
\text { subprocessos: aceitar a realidade, reconstrução } \\
\text { da esperança e pensamentos positivos } \\
\text { intencionais. A principal preocupação dos pais } \\
\text { é perder a esperança. }\end{array}$ & $\begin{array}{l}\text { Acesso a informações sobre a } \\
\text { saúde da criança. } \\
\text { Aumento da experiência e } \\
\text { conhecimento. } \\
\text { Conexão com os outros. } \\
\text { Estar no circuito. } \\
\text { Reafirmação da fé. }\end{array}$ \\
\hline $\begin{array}{l}\text { Popp, Conway \& } \\
\text { Pantaleao (18) } \\
2015 \\
\text { USA }\end{array}$ & $\begin{array}{l}\text { Parents' Experience } \\
\text { with Their Child's } \\
\text { Cancer Diagnosis: Do } \\
\text { Hopefulness, Family } \\
\text { Function, and Perceptions } \\
\text { of Care Matter? }\end{array}$ & $\begin{array}{l}\text { Distinguir as experiências dos pais } \\
\text { resolvidos e não resolvidos com o } \\
\text { diagnóstico de cancro dos seus filhos } \\
\text { em relação à esperança, adaptação } \\
\text { familiar e perceção de cuidados. }\end{array}$ & Quantitativo & $\begin{array}{l}\text { Pais não resolvidos com o diagnóstico clínico } \\
\text { dos seus filhos demonstram diminuição da } \\
\text { esperança e da capacidade de atuação perante } \\
\text { a doença. } \\
\text { Pais que conseguem conjugar a esperança } \\
\text { e a capacidade de ação perante a doença } \\
\text { conseguem traçar objetivos e um plano para } \\
\text { os atingir. }\end{array}$ & $\begin{array}{l}\text { Estabelecimento de objetivos e de } \\
\text { um plano para os atingir. }\end{array}$ \\
\hline $\begin{array}{l}\text { Kamihara, Nyborn, } \\
\text { Olcese, Nickerson } \\
\text { \& Mack }{ }^{(19)} \\
2015 \\
\text { EUA }\end{array}$ & \begin{tabular}{|l|} 
Parental Hope for \\
Children With Advanced \\
Cancer
\end{tabular} & $\begin{array}{l}\text { Identificar quais as expetativas dos pais } \\
\text { da criança com cancro em relação à } \\
\text { vivência de esperança. }\end{array}$ & Qualitativo & $\begin{array}{l}\text { Pais têm esperança na cura (88\%), numa } \\
\text { vida longa para os filhos (66\%), que o tumor } \\
\text { estabilize ou diminua com tratamento (47\%), } \\
\text { que o tratamento prolongue a vida (38\%), } \\
\text { qualidade de vida ( }(94 \%), \text { normalidade (88\%), } \\
\text { minimização do sofrimento }(75 \%) \text {, amor e } \\
\text { relações para os filhos (66\%), esperança por } \\
\text { parte de outros membros da família (28\%) e } \\
\text { esperança por melhor tratamento (25\%). }\end{array}$ & $\begin{array}{l}\text { Negação. } \\
\text { Prolongamento da vida. } \\
\text { Qualidade de vida. } \\
\text { Perceção de normalidade. } \\
\text { Sofrimento. } \\
\text { Estabelecimento de relações } \\
\text { significativas. }\end{array}$ \\
\hline $\begin{array}{l}\text { Borgestig, } \\
\text { Rytterström \& } \\
\text { Hemmingsson }{ }^{(20)} \\
2017 \\
\text { Sweden }\end{array}$ & $\begin{array}{l}\text { Gaze-based assistive } \\
\text { technology used in } \\
\text { daily life by children } \\
\text { with severe physical } \\
\text { impairments - parents' } \\
\text { experiences }\end{array}$ & $\begin{array}{l}\text { Descrever e explorar as experiências } \\
\text { dos pais quando os filhos com paralisia } \\
\text { cerebral recebem terapia guiada pelo } \\
\text { olhar. }\end{array}$ & \begin{tabular}{|l} 
Qualitativo: \\
Abordagem \\
Fenomenológica
\end{tabular} & $\begin{array}{l}\text { A terapia guiada pelo olhar conduz a um } \\
\text { aumento da esperança nos pais das crianças. A } \\
\text { esperança está relacionada com a altura em que } \\
\text { as crianças começam a utilizar esta tecnologia } \\
\text { e com a conceção de um futuro melhor para os } \\
\text { seus filhos. Os pais demonstram esperança de } \\
\text { que os outros compreendam os seus filhos e os } \\
\text { ajudem a desenvolver-se. }\end{array}$ & $\begin{array}{l}\text { Perceção de um futuro melhor para } \\
\text { a criança. }\end{array}$ \\
\hline $\begin{array}{l}\text { Illum, Bonderup \& } \\
\text { Gradel|(21) } \\
2018 \\
\text { Dinamarca }\end{array}$ & $\begin{array}{l}\text { Parents' Expressions } \\
\text { of Concerns and Hopes } \\
\text { for the } \\
\text { Future and Their } \\
\text { Concomitant } \\
\text { Assessments of Disability } \\
\text { in their Children }\end{array}$ & $\begin{array}{l}\text { Avaliar a capacidade dos pais de } \\
\text { expressar as suas preocupações e } \\
\text { esperança relativamente ao futuro dos } \\
\text { seus filhos com deficiência; } \\
\text { Avaliar a incapacidade dos seus filhos e } \\
\text { relacionar os dados obtidos. }\end{array}$ & Mixed Methods & $\begin{array}{l}\text { Os pais podem expressar dados válidos } \\
\text { e confiáveis sobre as suas preocupações } \\
\text { e esperança para o futuro e avaliar com } \\
\text { segurança a incapacidade dos seus filhos. } \\
\text { Preocupações mais frequentes: educação; } \\
\text { compreensão, boa vontade e comunicação } \\
\text { entre pais; e apoio da comunidade. } \\
\text { Os pais demonstraram esperança de que a } \\
\text { criança tenha uma boa vida futura. }\end{array}$ & $\begin{array}{l}\text { Preocupações sobre o futuro. } \\
\text { Perceção sobre a avaliação da } \\
\text { deficiência. }\end{array}$ \\
\hline
\end{tabular}

res relacionados com a criança, a saber: perceção ou preocupações sobre o futuro; progressáo da doença; presença de dor e sofrimento; qualidade de vida e bem-estar; negação; efeitos do tratamento; prolongamento da vida e estabelecimento de objetivos e de um plano para os atingir; medo e incerteza; suporte psicossocial; pensamento/foco num presente positivo; consciencialização para os aspetos positivos ou negativos relacionados com a doença da criança.

No que diz respeito aos fatores relacionados com os pais, os estudos identificam os seguintes: ansiedade, depressão e bem-estar; perceção de apoio; estabelecimento de relaçóes significativas; espiritualidade; sobrecarga de informação; esgotamento físico e emocional. Esta perspetiva encontra-se integrada na função de papel, pelo que os fatores relacionados com o desempenho do papel parental parecem ser condicionantes das vivências de esperança. A este nível foi valorizado pelos pais, a comunicaçấo estabelecida com os profissionais de saúde; a informação e /ou conhecimento sobre a doença e tratamentos a que a criança vai ser submetida; participação dos pais nos cuidados à criança; sentido de controle; confiança e satisfação com os cuidados recebidos; perceção de normalidade.

Apesar da entrevista ter sido a técnica de recolha de dados utilizada na maior parte dos estudos em análise, destacamos o recurso dos dois estudos de natureza quantitativa a instrumentos de avaliação da esperança. Nestes foram aplicados dois instrumentos de avaliação distintos: "The Trait Hope Scale" que é constituída por 12 itens específicos que após preenchidos resultam num score oscilante entre 12 e 96; ${ }^{(22)}$ e a "Herth Hope Index" constituída também por 12 itens, no entanto, o seu score varia entre 12 e 48, “(...) sendo que quanto maior o escore, mais alto o nível de esperança". ${ }^{(23)}$

Ambos os instrumentos avaliam a esperança acerca do futuro na pessoa adulta. Atendendo ao objetivo 
da revisão identificaram-se itens que se constituem como fatores influenciadores da esperança nos estudos analisados: perceçáo de alcançar objetivos traçados e/ou cansaço, e preocupação acerca do futuro.

\section{Discussão}

A evidência da pesquisa realizada e a consequente aplicabilidade dos seus resultados, permitiu uma maior compreensão do conceito esperança, constatando-se que na grande maioria dos artigos é definida como positiva e motivadora. ${ }^{(9,15,21)}$ É também descrita como essencial, poderosa, deliberada, que sustenta a vida, dinâmica e encarada como um processo cíclico, influenciada pelo tempo. ${ }^{(9)} \mathrm{O}$ mesmo autor acrescenta que é fortalecedora e fornece orientação interna, preparando para os desafios e para o pior que poderá, eventualmente, acontecer. ${ }^{(9)}$ A esperança é um recurso interno e motivacional que orienta para o presente e futuro. Por outro lado, ajuda os pais no processo de coping em relação à doença do filho, mesmo que exista um mau prognóstico ou possibilidade de morte. ${ }^{(15)}$ Esta vai contribuir de forma decisiva para a sua preparação para aspetos negativos tanto da doença como do tratamento. ${ }^{(15)}$ A esperança oscila ao longo do tempo em funçáo do contexto psicossocial em que os pais se inserem, e é influenciada pelas características pessoais, pelos recursos externos e pela resposta do filho ao tratamento. ${ }^{(15-16)}$

Num estudo que se debruça sobre pais de crianças com o diagnóstico de cancro, é concluído que os pais resolvidos com o diagnóstico dos filhos apresentam um fator de resiliência facilitador do processo de coping. ${ }^{(18)}$ Por outro lado, os que não se encontram resolvidos, demonstram uma diminuição da esperança e da capacidade de atuação perante a doença. ${ }^{(18)}$ Pais que conseguem conjugar a esperança e capacidade de ação perante a doença traçam objetivos e delineiam um plano para os atingir. ${ }^{(18)}$ Em outro estudo também relacionado com crianças com cancro, os autores referem que um dos fatores que influência a tomada de decisão dos pais em relação ao tratamento, e aos cuidados à criança, é a esperança parental. ${ }^{(17)}$ Também numa investigação referente a de crianças com deficiência intelectual (Síndrome de Down, Paralisia Cerebral e Deficiência
Intelectual de Etiologia Inespecífica), a esperança foi medida como uma meta face a um comportamento dirigido, onde o pensamento baseado em agência (motivação necessária para utilizar rotas que levem a objetivos desejados), foi interpretado como um fator de resiliência e de bem-estar psicológico para os pais. ${ }^{(14)}$

O caráter cíclico da esperança ao longo do tempo é justificado numa investigação realizada a 35 pais de crianças com o diagnóstico de cancro, com recurso a três entrevistas diferentes, emergindo duas categorias distintas: esperança orientada para o futuro e orientada para o presente. ${ }^{(15)}$ Dentro destas categorias foram definidos temas específicos. ${ }^{(15)}$ Para a esperança orientada para o futuro o enunciado pelos pais foi: esperança pela cura dos filhos, por um futuro, por um milagre e por mais tempo de qualidade com a criança. ${ }^{(15)} \mathrm{A}$ esperança orientada para o presente tem como subcategorias os filhos não terem sofrimento nem complicaçôes. ${ }^{(15)}$ Além do enunciado concluíram que esta muda ao longo do primeiro ano após o diagnóstico da doença. ${ }^{(15)} \mathrm{A}$ esperança parental também é caracterizada como uma força de vida dinâmica, multidimensional e orientação para a vida e para o futuro, perante a gestáo dos cuidados de saúde aos filhos. ${ }^{(6)}$

Outra característica deste conceito é ser um processo construtivo. ${ }^{(9)}$ Num estudo realizado com 16 pais de crianças com cancro, com recurso a entrevistas com perguntas abertas, os autores concluíram que o processo de esperança passa por quatro subprocessos: aceitar a realidade, estabilização, reconstrução da esperança e pensamentos positivos intencionais. ${ }^{(9)} \mathrm{O}$ aceitar a realidade consiste nos pais sentirem que necessitam de aceitar o diagnóstico do filho e a lentidão inerente ao processo de tratamento. A segunda fase prende-se com a estabilização, proporcionando algum controlo, avaliando as circunstâncias e gerindo as necessidades de cuidados. Sucede-se a reconstrução de esperança que motiva os pais a esperarem o melhor e a resolver os seus medos de perder a esperança. $\mathrm{O}$ último passo descrito são os pensamentos positivos intencionais que passam por encontrar um lado positivo na vivência. ${ }^{(9)}$

Apesar de ser um processo e vivência individual e dinâmica existem fatores que influenciam a esperança parental como a avaliaçáo de saúde dos filhos, o ganho de experiência, o conhecimento em relaçáo à doença, o contato com os outros, o estar a par do cir- 
cuito de tratamentos e a reafirmação de fé. ${ }^{(9)}$ Num estudo que desenvolvido com 35 pais de crianças com o diagnóstico de cancro, com recurso a entrevista, os investigadores identificaram facilitadores e barreiras à manutenção da esperança. ${ }^{(18)} \mathrm{A}$ consciência parental sobre a evolução positiva da doença e efeitos dos tratamentos dos filhos, o foco nas variáveis positivas do presente, a espiritualidade/religiáo e o suporte social foram nomeados como facilitadores à manutenção da esperança enquanto que a consciência parental acerca da evolução negativa da doença e dos tratamentos, a sobrecarga de informação por parte dos profissionais de saúde, o negativismo das outras pessoas próximas, o esgotamento físico e emocional e os medos e incertezas constituíram-se barreiras à continuidade de esperança. ${ }^{(16)}$ Estes resultados vêm corroborar o descrito na literatura científica mais recente. ${ }^{(24-26)}$

O foco nas variáveis positivas do presente é abordado também num estudo que comtempla 11 pais de crianças com paralisia cerebral que são alvo de terapia guiada pelo olhar. ${ }^{(20)}$ Os autores constataram que a esperança é influenciada pela altura em que os filhos começam a utilizar esta tecnologia, relacionando-a com uma conceção de futuro para estas crianças. ${ }^{(20)}$

A esperança parental, de modo geral, está relacionada com a cura dos filhos e com um futuro melhor, ${ }^{(16-17,19,25)}$ sendo predominantemente nos pais a esperança pela cura, seguida da possibilidade de uma vida longa e da estabilização da doença com o tratamento. ${ }^{(19)}$ Acrescenta-se ainda a esperança relacionada com a qualidade de vida, com a normalidade, com a minimizaçáo do sofrimento e num melhor tratamento. ${ }^{(19)}$ Quando não existe por parte dos pais esperança na cura, estes encontram outros motivos para mantê-la. ${ }^{(15)}$ A principal preocupação dos pais, passa pela possibilidade de perder a esperança, sendo que esta é encarada como um processo social básico. ${ }^{(9)}$

Numa investigação realizada a 119 pais de crianças com espinha bífida, atrofias musculares espinhais, distúrbios musculares, paralisia cerebral e diversas deficiências, concluiu-se que estes expressam preocupaçôes relacionadas com a qualidade dos cuidados prestados. Estas incluem a compreensão das necessidades da família, apoio social, estratégias de enfrentamento e envolvimento nas decisóes. ${ }^{(21)}$ A sua esperança está relacionada com qualidade de vida, bem-estar e saúde futura, educação, assistência médica e apoio de profissionais de saúde. ${ }^{(21)} \mathrm{O}$ mesmo estudo relacionou a gravidade da incapacidade dos filhos, com as preocupaçóes e com a esperança livremente expressas. ${ }^{(21)}$ Concluiu-se que a preocupação de que a criança náo sinta satisfação com a vida adulta é mais vezes manifestada pelos pais de crianças com deficiências mais graves, que mantêm a esperança que os filhos aceitem a sua própria deficiência e tenham autoconfiança. ${ }^{(21)}$

Os profissionais de saúde tornam-se fundamentais na promoção de esperança apesar de muitas vezes os clínicos terem dificuldade em abordar este tema, algumas das vezes com receio do prognóstico da doença ser contraditório à promoção da esperança. (19) Estes necessitam de identificar os estádios e perceçôes relativas ao tema forma a perceber e guiar os pais nas suas vivências de esperança. ${ }^{(15)}$ Além disso a comunicação eficaz por parte dos profissionais de saúde é imperativa pois atua como fator promotor. ${ }^{(17)}$

\section{Conclusão}

Do resultado da análise dos estudos que constituíram esta pesquisa, emergiram diversos factos que permitiram evidenciar o que influencia a vivência de esperança nos pais de crianças com doença crónica, permitindo dar resposta à questáo inicialmente levantada. Contatou-se que a esperança é multidimensional devido a ter várias características como ser poderosa, essencial à vida, cíclica ao longo do tempo, fornecedora de orientação interna. É encarada pela maioria dos pais como um recurso interno fortalecedor. A esperança associada à aceitação do diagnóstico assume-se como essencial para o traçar de objetivos e posteriormente para a elaboração de um plano sensato que conduza à concretização destes. É influenciada por uma serie de fatores destacando-se a resposta da criança ao tratamento e o suporte psicossocial da família. Algumas das estratégias promotoras de esperança são: a consciência positiva sobre a doença e tratamentos, o suporte social adequado à família, o recurso à espiritualidade, o ganho de experiência e domínio em relaçáo aos tratamentos do filho e o foco nos fatores positivos do presente. As barreiras/fatores de ameaça à esperança identificados são: consciência negativa sobre a situação atual dos filhos em relação ao estado de saú- 
de e tratamentos, sobrecarga de informação, cansaço/ esgotamento físico e emocional dos pais e pensamentos negativos das pessoas que os rodeiam. Os profissionais de saúde e em particular os enfermeiros, ao terem em conta na sua prática profissional os fatores promotores ou ameaçadores de esperança, podem encontrar inúmeras oportunidades para promover esperança parental. A continuidade da investigação acerca destes fatores, permitirão melhorar a acurácia dos diagnósticos de enfermagem, assim como das intervençóes promotoras de esperança parental. A necessidade de mais estudos de investigação foi demonstrada com a constatação da produção continua de conhecimento. Com o supracitado é possível concluir que este é um tema emergente, com implicaçóes diretas nos cuidados de saúde e que necessita de atualizaçáo constante para uma melhoria da disciplina e da prática de enfermagem. As limitaçóes descritas na maior parte dos estudos constituíram a impossibilidade de generalização para toda a população devido a serem amostras pouco representativas em relaçáo à globalidade (quer pelo seu tamanho, quer pela especificidade de algumas patologias) o que constitui uma fragilidade a esta revisão sistemática da literatura.

\section{Referências}

1. Mendes MG, Martins MM. Parceria nos cuidados de enfermagem em pediatria: do discurso à ação dos enfermeiros. Rev Enferm Ref. 2012;3(6):113-21.

2. World Health Organization (WHO). Preventing chronic diseases. Geneva: WHO; 2018 [cited 2020 Oct 7]. Available from: https://www. who.int/chp/chronic_disease_report/contents/part1.pdf?ua=1

3. Cutcliffe JR, Herth $K$. The concept of hope in nursing 1: its origins, background and nature. Br J Nurs. 2002;11(12):832-40.

4. Kylmä J, Vehviläinen-Julkunen K. Hope in nursing research: a metaanalysis of the ontological and epistemological foundations of research on hope. J Adv Nurs. 1997;25(2):364-71.

5. Farran CJ, Wilken C, Popovich JM. Clinical assessment of hope. Issues Ment Health Nurs. 1992;13(2):129-38.

6. Samson A, Tomiak E, Dimillo J, Lavigne R, Miles S, Choquette M, et al. The lived experience of hope among parents of a child with Duchenne muscular dystrophy: perceiving the human being beyond the illness. Chronic IIIn. 2009;5(2):103-14.

7. Cavaco VS, José HM, Louro SP, Ludgero AF, Martins AF, Santos MC. Qual o papel da esperança na saúde da pessoa? - Revisão Sistemática. Rev Refer. 2010;2(12):93-103.

8. Charepe ZC. Promover a esperança em pais de crianças com doença crónica - modelo de intervenção em ajuda mútua. Lisboa: Universidade Católica Portuguesa; 2014.176 p.
9. Bally JM, Duggleby W, Holtslander L, Mpofu C, Spurr S, Thomas R, et al. Keeping hope possible: a grounded theory study of the hope experience of parental caregivers who have children in treatment for cancer. Cancer Nurs. 2014;37(5):363-72.

10. Sisk BA, Kang TI, Mack JW. Sources of parental hope in pediatric oncology. Pediatr Blood Cancer. 2018;65(6):e26981.

11. Joanna Briggs Institute. Joanna Briggs Institute Reviewers' Manual: 2014 ed. Australia: The Joanna Briggs Institute; 2014.

12. Peters M, Godfrey C, Mcinerney P, Baldini C, Khalil H, Parker D. Guidance for the Conduct of JBI Scoping Reviews. In: Aromataris E, Munn Z, editors. Joana Briggs Institute Reviewer's Manual. Australia: The Joanna Briggs Institute; 2017. Chapter: 11.

13. Moher D, Liberati A, Tetzlaff J, Altman DG; PRISMA Group. Preferred reporting items for systematic reviews and meta-analyses: the PRISMA statement. PLoS Med. 2009;6(7):e1000097.

14. Lloyd TJ, Hastings R. Hope as a psychological resilience factor in mothers and fathers of children with intellectual disabilities. J Intellect Disabil Res. 2009;53(12):957-68.

15. Granek L, Barrera M, Shaheed J, Nicholas D, Beaune L, D'Agostino N, et al. Trajectory of parental hope when a child has difficult-to-treat cancer: a prospective qualitative study. Psychooncology. 2013;22(11):2436-44.

16. Barrera M, Granek L, Shaheed J, Nicholas D, Beaune L, D’Agostino $\mathrm{NM}$, et al. The tenacity and tenuousness of hope: parental experiences of hope when their child has a poor cancer prognosis. Cancer Nurs. 2013;36(5):408-16.

17. Markward MJ, Benner K, Freese R. perspectives of parents on making decisions about the care and treatment of a child with cancer: a review of literature. Am Psychol Association. 2013;31(4):406-13.

18. Popp JM, Conway M, Pantaleao A. Parents' experience with their child's cancer diagnosis: do hopefulness, family functioning, and perceptions of care matter? J Pediatr Oncol Nurs. 2015;32(4):253-60.

19. Kamihara J, Nyborn JA, Olcese ME, Nickerson T, Mack JW. Parental hope for children with advanced cancer. Pediatrics. 2015;135(5):868-74.

20. Borgestig M, Rytterström P, Hemmingsson H. Gaze-based assistive technology used in daily life by children with severe physical impairments - parents' experiences. Dev Neurorehabil. 2017;20(5):301-8.

21. Illum NO, Bonderup M, Gradel KO. Parents' expressions of concerns and hopes for the future and their concomitant assessments of disability in their children. Clin Med Insights Pediatr. 2018;12:1179556518784948.

22. Mednick L, Cogen F, Henderson C, Rohrbeck C, Kitessa D, Streisand R. Hope More, Worry Less: Hope as a Potential Resilience Factor in Mothers of Very Young Children with Type 1 Diabetes. Child Health Care. 2007;36(4):385-96.

23. Sartore AC, Grossi SA. Escala de Esperança de Herth-instrumento adaptado e validado para a língua portuguesa. Rev Esc Enferm USP. 2008;42(2):227-32.

24. Cless JD, Nelson Goff BS, Durtschi JA. Hope, coping, and relationship quality in mothers of children with down syndrome. J Marital Fam Ther. 2018;44(2):307-22.

25. Finke EH, Kremkow JM, Drager KD, Murillo A, Richardson L, Serpentine EC. "I Would Like for My Child to be Happy with His Life": Parental hopes for their children with ASD across the lifespan. J Autism Dev Disord. 2019;49(5):2049-68.

26. Davidson Arad B, McLeigh JD, Katz C. Perceived Collective Efficacy and Parenting Competence: The Roles of Quality of Life and Hope. Fam Process. 2020;59(1):273-87. 